Class III is made by combining the method of Class I with that of Class II as shown in Fig. 30. One element (and naturally all of its fellows in the group) runs diagonally while the other element is placed by knight's moves. There is consequently a choice of two elements, either of which may dominate the diagonals or be located by knight's moves. In the case shown in Fig. 30 it is evident that " $a$ " may occupy the cells marked " $a$ " or those marked " $a$," there being eight possible knight's moves from any cell. This fact gives still another choice of two different ways, so there is a choice of four methods in Class III.

Summarizing the foregoing results:

For the basic row there is a choice of 14400 .

$$
\begin{array}{r}
\text { For Class I squares } 2 \times 14400=28800 \\
\text { For Class II squares } 2 \times 14400=28800 \\
\text { For Class III squares } 4 \times 14400=57600 \\
\frac{I 15200 .}{}
\end{array}
$$

So there are at least II 5200 different ways in which a perfect $5 \times 5$ square may be made.

According to the figures herein given the number of variations of the different sizes of squares that have been considered increases as follows:

$$
\begin{array}{lrr}
3 \times 3 & \text { square } & 1 \\
4 \times 4 & \text { " } & 4,352 \\
5 \times 5 & & 115,200
\end{array}
$$

\title{
NOTES ON NUMBER SERIES USED IN THE CONSTRUCTION OF MAGIC SQUARES.
}

It has long been known that magic squares may be constructed from a series of numbers which do not progress in arithmetical order. Experiment will show, however, that any haphazard series cannot be used for this purpose, but that a definite order of sequence is necessary which will entail certain relationships between different members of the series. It will therefore be our endeavor in the present article to determine these relationships and express the same in definite terms.

Let Fig. 3I represent a magic square of $4 \times 4$. By rule No. 4 in the "New Analysis of Magic Squares" it is seen that "the sum of the terms in any two contiguous corner cells is equal to the sum of 
the terms in the two middle cells in the opposite outside column." Therefore, in Fig. 3I, $a+d=v+s$, and it therefore follows that $a-v=s-d$. In other words, these four quantities form a group with the interrelationship as shown. By the same rule (No. 4) it is also seen that $a+t=l+p$, and hence also, $a-l=p-t$, giving another group of four numbers having the same form of interrelationship, and since both groups have " $a$ " as an initial number, it is evident that the increment used in one of these groups must be different from that used in the other, or duplicate numbers would result. It therefore follows that the numbers composing a magic square are not made up of a single group, but necessarily of more than one group.

Since we have seen that the term " $a$ " forms a part of two groups, we may write both groups as shown in Fig. 32, one horizontally and the other perpendicularly.

Next, by rule No. 5, it is shown that "the sum of the two end

\begin{tabular}{|l|l|l|l|}
\hline$a$ & $b$ & $c$ & $d$ \\
\hline$g$ & $h$ & $k$ & $l$ \\
\hline$m$ & $m$ & $o$ & $p$ \\
\hline$t$ & $r$ & $s$ & $y$ \\
\hline
\end{tabular}

Fig. 31.

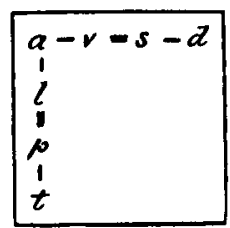

Fig. 32.

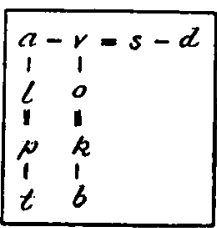

Fig. 33 .

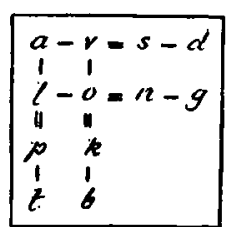

Fig. 34.

terms of any inside column (either horizontal or perpendicular) is equal to the sum of the two middle terms in the other parallel column." It therefore follows that $v+b=k+o$ or $v-o=k-b$. Using the term $v$ as the initial number, we write this series perpendicularly as shown in Fig. 33. In the same way it is seen that $l+g=n+o$, or $l-o=n-g$, thus forming the second horizontal column in the square (Fig. 34). Next $p+m=h+k$ or $p-k=h-m$, forming the third horizontal column and in this simple manner the square may be completed as shown in Fig. 35.

It is therefore evident that a $4 \times 4$ magic square may be formed of any series of numbers whose interrelations are such as to permit them to be placed as shown in Fig. 35 .

The numbers $I$ to 16 may be so placed in a great variety of ways, but the fact must not be lost sight of that, as far as the construction of magic squares is concerned, they only incidentally possess the quality of being a single series in straight arithmetical 
order, being really composed of as many groups as there are cells in a column of the square. Unless this fact is remembered, a clear conception of the quantities of the series cannot be formed.

In illustration of the above remarks, three diagrams are given in Figs. 36,37 and 38 . Figs. $3^{6}$ and 37 show arrangements of the numbers $I$ to 16 from which the diverse squares Figs. 39 and 40 are formed by the usual method of construction.

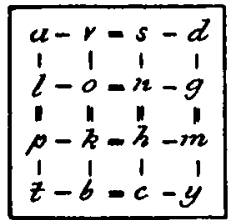

Fig. 35.

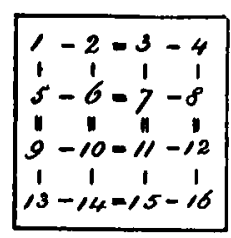

Fig. 36.

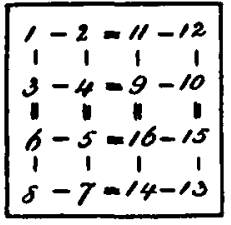

Fig. 37.

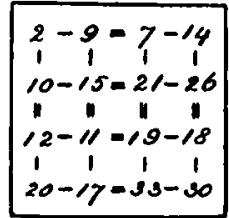

Fig. 38.

Fig. $3^{8}$ shows the arrangement of an irregular series of sixteen numbers, which, when placed in the order of magnitude run as follows :

$$
\text { 2-7-9-10- I I- I 2-I 4- I 5- I 7- I 8-1 9-20-2 I-26-30-33 }
$$

The magic square formed from this series is given in Fig. 41.

In the study of these number series the natural question presents itself: Can as many diverse squares be formed from one series as from another? This question opens up a wide and but little explored region as to the diverse constitution of magic squares. This

\begin{tabular}{|c|c|c|c|}
\hline 1 & 14 & 15 & 4 \\
\hline 8 & 11 & 10 & 5 \\
\hline 12 & 7 & 6 & 9 \\
\hline 13 & 2 & 3 & 16 \\
\hline
\end{tabular}

Fig. 39.

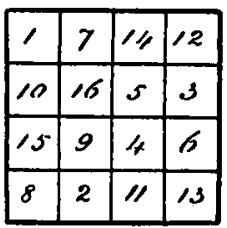

Fig. 40.

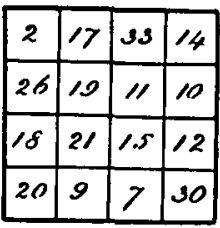

Fig. 4I.

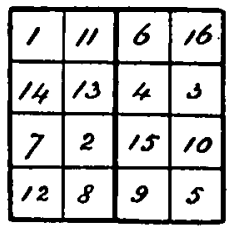

Fig. 42.

1dea can therefore be merely touched upon in the present article, examples of several different plans of construction being given in illustration and the field left at present to other explorers.

Three examples will be given, the first being what is sometimes termed a "perfect" square, or one in which any two numbers that are geometrically opposite and equidistant from the center of the square will be equal in summation to any other pair of numbers so 
situated. The second example will be a square in which the sum of every diagonal of the four sub-squares of $2 \times 2$ is equal, and the third example will be a square in which the pairs of numbers having similar summations are arranged symmetrically in relation to a perpendicular line through the center of the square. Figs. 39, 40 and 42 illustrate these three examples of squares.

Returning now to the question previously given, but little rcflection is required to show that it must be answered in the negative for the following reasons. Fig. $4 \mathrm{I}$ represents a magic square having

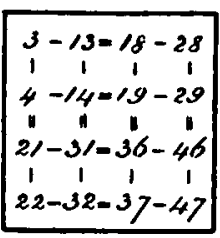

Fig. 4I.

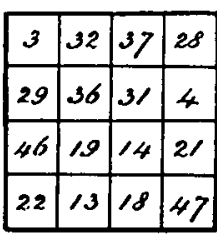

Fig. 42.

no special qualities excepting that the columns, horizontal, perpendicular and diagonal all have the same summation, viz., 66. Hence any series of numbers that can be arranged as shown in Fig. 35 will yield magic squares as outlined. But that it shall also produce squares having the qualifications that are termed "perfect," may or may not be the case accordingly as the series may or may not be capable of still further arrangement.

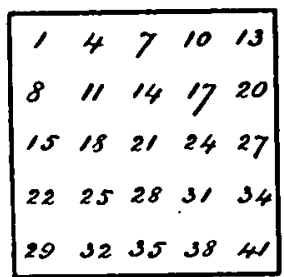

Fig. 43 .

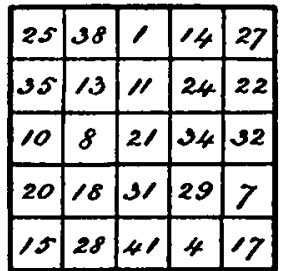

Fig. 44

Referring to Fig. 3 I, if we amend our definition by now calling it a "perfect" square, we shall at once introduce the following continuous equation:

$$
a+y=h+o=t+d=n+k=b+s=c+v=g+p=m+l,
$$

and if we make our diagram of magic square prriducing numbers conform to these new requirements, the number of groups will at once be greatly curtailed. 
The multiplicity of algebraical signs necessary in our amended diagram is so great that it can only be studied in detail, the complete diagram being a network of minus and equality signs.

The result will therefore only be given here, formulated in the following laws which apply in large measure to all "perfect" squares.

I. Perfect magic squares are made of as many series or groups of numbers as there are cells in a column.

II. Each series or group is composed of as many numbers as there are groups.

III. The differences between any two adjoining numbers of a series must obtain between the corresponding numbers of all the series.

IV. The initial terms of the series compose another series, as do the second, third, fourth terms and so on.

$\mathrm{V}$. The differences between any adjoining numbers of these secondary series must also obtain between the corresponding terms of all the secondary series.

The foregoing rules may be illustrated by the series and perfect square shown in Figs. 36 and 39.

Following and consequent upon the foregoing interrelations of these numbers is the remarkable quality possessed by the "perfect" magic square producing series as follows:

If the entire series is written out in the order of magnitude and the differences between the adjacent numbers are written below, the row of differences will be found to be geometrically arranged on each side of the center as will be seen in the following series taken from Fig. 43.

$$
\begin{aligned}
& 3 \text { - } 4 \text {-I3-I4-I8-I9-2I-22-28-29-3I-32-36-37-46-47 }
\end{aligned}
$$

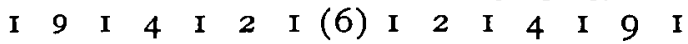

In the above example the number 6 occupies the center and the other numbers are arranged in geometrical order on each side of it. It is the belief of the writer that this rule applies to all "perfect" squares whether odd or even.

The following example will suffice to illustrate the rule as applied to a $5 \times 5$ magic square, Fig. 45 showing the series and Fig. 46 the square.

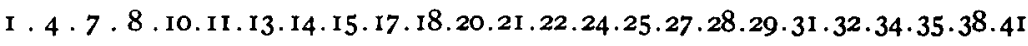

\begin{tabular}{llllllllllll|llllllllllll}
3 & 3 & I & 2 & I & 2 & I & I & 2 & I & 2 & I & I & 2 & I & 2 & I & I & 2 & I & 2 & I & 3 & 3
\end{tabular}

L. S. Frierson.

Frierson, LA. 